\title{
Adaptación de cofias Cobalto-Cromo confeccionadas con dos técnicas de colado sobre dos líneas de terminación
}

Fit of Cobalt-Chromium alloy copings fabricated with two casting techniques on two termination lines.

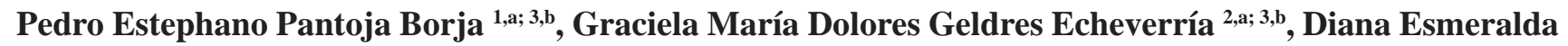
Castillo Andamayo ${ }^{\text {3a,b,c; ;d }}$, Martín Gilberto Quintana del Solar ${ }^{3, a, b, d ; ; d ~}$

\section{RESUMEN}

Las técnicas de cera perdida colado por centrifugación convencional, son técnicas vigentes para obtener cofias de Cobalto-Cromo (Co-Cr) para coronas metalcerámica. Objetivo: Evaluar in vitro, la adaptación marginal e interna de cofias unitarias de aleación Co-Cr, realizadas sobre dos líneas de terminación: chamfer y bisel, fabricadas con las técnicas de cera perdida colada por centrifugación convencional (CPCC) y por inducción (CPCI). Materiales y métodos: Se evaluó la adaptación marginal e interna de 13 cofias unitarias de aleación Co-Cr para cada técnica y línea de terminación, usando la técnica de réplica de silicona. Las muestras fueron divididas en sentido vestíbulo-palatino y en sentido mesio-distal, observadas en un estéreomicroscopio a un aumento de 40X y se evaluó la zona cervical, axial y oclusal. Resultados: Los análisis estadísticos incluyeron la prueba $t$ de Student y U Mann-Whitney, previamente se evaluó los supuestos de normalidad con la prueba de Shapiro-Wilk para analizar diferencias estadísticamente significativas entre los grupos $(\mathrm{p}<0.05)$. La discrepancia marginal fue menor a $120 \mu \mathrm{m}$ y la discrepancia interna menor a $350 \mu \mathrm{m}$ para ambas líneas de terminación y técnica de colado. Conclusión: Los resultados en la adaptación marginal e interna de cofias con líneas de terminación chamfer y bisel fabricadas con las técnicas de cera perdida colada por centrifugación convencional e inducción, los valores descriptivos no fueron concluyentes, ya que en la mayoría de los puntos evaluados no existió diferencia estadísticamente significativa. Además en las dos líneas de terminación chamfer y bisel se encontraron rangos clínicamente aceptables.

PALABRAS CLAVES: Adaptación, aleaciones de cromo, ceras.

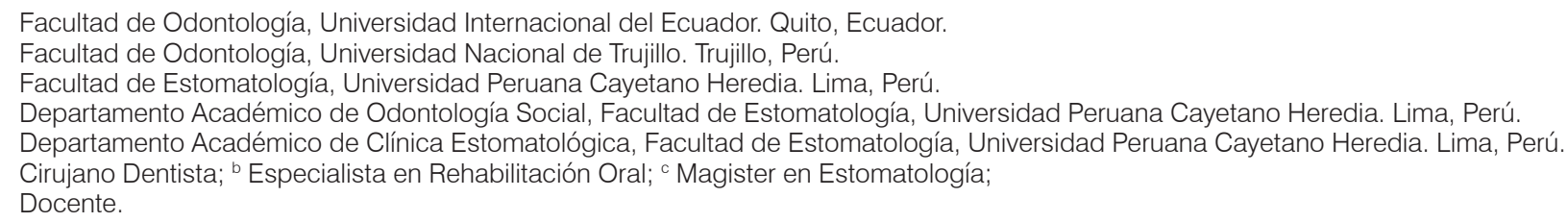




\section{SUMMARY}

The techniques of lost wax cast by conventional centrifugation are current techniques to obtain Cobalt-Chrome (Co-Cr) copings for metalceramic crowns. Objective: Evaluate in vitro, the marginal and internal adaptation in unitary Co-Cr alloy copes, made on two termination lines: chamfer and bevel, manufactured with the techniques of lost wax cast by conventional centrifugation (CPCC) and by induction (CPCI), according to type of cut, area and measurement points. Materials and methods: The marginal and internal adaptation of 13 co-units of Co-Cr alloy for each technique and termination line was evaluated. The silicone replication technique was used. The samples were divided in the vestibulo-palatal direction and in the mesio-distal direction, observed in a stereomicroscope at a 40X magnification and the cervical, axial and occlusal areas were evaluated. Results: Statistical analyzes included the Student's t-test and U Mann-Whitney, previously the assumptions of normality were evaluated with the Shapiro-Wilk test to analyze statistically significant differences between the groups $(\mathrm{p}<0.05)$. The marginal discrepancy was less than $120 \mu \mathrm{m}$ and the internal discrepancy less than $350 \mu \mathrm{m}$. Conclusion: The results in the marginal and internal adaptation of copings with chamfer termination lines and bevel fabricated with the techniques of lost wax cast by conventional centrifugation and induction were not conclusive to determine which technique is the best since in most of the points evaluated there was not a statistically significant difference. Also in the two lines of termination cnamfer and bevel, there were found clinical acceptable ranges.

\section{KEYWORDS: Adaptation, chromium alloys, waxes.}

\section{INTRODUCCIÓN}

Las coronas metalcerámica, son una alternativa de tratamiento exitoso con una tasa de supervivencia por más de diez años (1). Uno de los factores más importantes del éxito a largo plazo es una adaptación marginal e interna adecuada; la falla de ello, conlleva a un fracaso en el tratamiento por: acúmulo de placa bacteriana y como resultado, mayor riesgo de lesiones cariosas o enfermedad periodontal y a su vez, la disolución del cemento (2). La adaptación marginal en prótesis fija, se define como "la exactitud con que la restauración encaja sobre una línea de terminación”.

El objetivo es lograr una unión íntima y adecuada entre la restauración y la preparación dentaria $(1,3)$. La distancia vertical que existe, entre la línea cervical de la preparación dentaria hasta el margen cervical de la corona, se define como discrepancia marginal (4).

La adaptación interna, se define como "el ajuste que se da entre la superficie del pilar dentario y la estructura de la restauración". La distancia perpendicular entre la restauración y la superficie de la preparación dentaria, se define como discrepancia interna. Esta distancia proporciona un espacio adecuado para el agente cementante, que debe ser uniforme para brindar retención y resistencia adecuada $(3,4)$. Huang et al., refieren que la Asociación Dental Americana, es- tablece un grosor máximo de $25 \mu \mathrm{m}$ para cemento de fosfato de zinc tipo I. Sin embargo, este valor, es muy difícil de conseguir y va a depender de la viscosidad del cemento que se utilice; así como también de la técnica de fabricación de la cofia (5). Por ello, varios autores concluyen que el valor de discrepancia marginal, para ser aceptado clínicamente, no debe exceder a $120 \mu \mathrm{m}(5,6)$ y sí nos referimos a discrepancia interna entre 50 y $350 \mu \mathrm{m}(7,8)$.

Las líneas de terminación para coronas metalcerámica más usadas, son chamfer y bisel (9). La de bisel, es donde la unión de la pared gingival y axial forma un ángulo de $135^{\circ}$ y la de chamfer, es donde la unión de la pared gingival y la axial dibujan un segmento curvo (10). Bishop K et al., concluyeron en un estudio, que la terminación en bisel es más conservadora y reduce el riesgo de daño pulpar, a comparación del chamfer (11). Y por otro lado, Bottino et al., encontró que la línea de terminación en chamfer presentaba una mejor adaptación marginal (1). Actualmente, existen varios métodos para la confección de las cofias en aleación Co-Cr. Entre ellas están la técnica de CPCC (soplete) y CPCI (eléctrico). También las técnicas de diseño y fabricación asistido por computadora (CAD/ CAM) sustractivos y aditivos $(12,13,14)$.

La técnica de cera pérdida colado por centrifugación convencional (CPCC), consiste en rodear el patrón de 
cera, con un material refractario resistente al calor. Luego la cera es evaporada en el horno de eliminación de cera, para después proceder a la fundición del metal base, empleando un soplete que funciona mediante oxígeno y gas, el cual produce una llama, que transforma la aleación de su estado sólido a líquido; luego se impulsa el metal fundido en el molde, a través del bebedero mediante una fuerza centrífuga (15).

La técnica de cera pérdida colada por centrifugación por inducción (CPCI), tiene el mismo procedimiento de CPCC, pero no es necesario el empleo del soplete para generar calor, pues la aleación se funde rápidamente en un crisol calentado por inducción eléctrica. El calor se genera por el sistema ánodo-cátodo, donde las cargas de la aleación son atraídas opuestamente, creando una desestabilización molecular, que induce al ascenso de la temperatura y al respectivo proceso de licuefacción de la aleación, para convertir la aleación a estado líquido por medio de altas temperaturas, que corresponden a los puntos de fusión de las aleaciones involucradas. Finalmente utiliza la fuerza centrífuga para impulsar la aleación por el bebedero (16). Es un método que si bien requiere mayor inversión, es un proceso controlado muy preciso, que es usado con aleaciones de cualquier tipo, permitiendo un excelente control de la temperatura del colado (17). Existen diferentes estudios que comparan las diversas técnicas de confección de cofias $\mathrm{Co}-\mathrm{Cr}$ y su efecto sobre la adaptación marginal. Abad et al., evaluaron la adaptación marginal con cuatro técnicas de colado convencionales: presión vacío y colado por inducción Nautilius ${ }^{\circledR}(\mathrm{BEGO} \mathrm{GmbH}$, Bremen, Alemania), centrifugado eléctrico y colado por inducción Fornax ${ }^{\circledR}$ (BEGO GmbH, Bremen, Alemania), por centrifugado eléctrico y fundición manual Fundor $\mathrm{T}{ }^{\circledR}$ (BEGO GmbH, Bremen, Alemania), y centrifugado por cuerda y colado manual Kerr ${ }^{\circledR}$ (Kerr Corporation, California, USA). Los resultados encontrados fueron: $59.26 \mu \mathrm{m}$ para Nautilius ${ }^{\circledR}, 63.6 \mu \mathrm{m}$ para Fornax ${ }^{\circledR}, 63.8 \mu \mathrm{m}$ para Fundor $\mathrm{T}{ }^{\circledR}$ y $69.08 \mu \mathrm{m}$ para Kerr®. Todos los sistemas mostraron rangos menores a $120 \mu \mathrm{m}$, pero la técnica de colado por inducción y presión al vació, mostro mejores resultados (17)

Gómez-Cogolludo et al., compararon ambas técnicas de colado convencional e inducción con cuatro tipos de aleaciones: paladio-oro, niquel-cromo-titanio, niquel-cromo (Ni-Cr), y titanio. Encontraron que el colado convencional, ofreció mejores valores de adaptación en cofias de paladio-oro, mientras que para niquel-cromo-titanio, no hubo diferencia significativa. Sin embargo, sugirieron tener en cuenta que el éxito de la técnica de soplete depende mucho de la experiencia del operador, mientras que para la técnica de inducción el control de la temperatura, es mucho más objetiva (18). Pulido et al., compararon la adaptación de cofias de Co-Cr y Ni-Cr, usando la técnica de colado por inducción eléctrica Fornax ${ }^{\circledR}(\mathrm{BEGO}$ $\mathrm{GmbH}$, Bremen, Alemania). Concluyeron que la adaptación de la aleación Co-Cr fue significativamente menor, que la aleación $\mathrm{Ni}-\mathrm{Cr}$ (4). El propósito de este estudio fue evaluar adaptación marginal e interna de cofias unitarias de aleación Co-Cr fabricadas con las técnicas CPCC y CPCI sobre dos líneas de terminación: chamfer y bisel.

\section{MATERIAL Y MÉTODOS}

El diseño de estudio fue de tipo experimental invitro y de corte transversal. Se seleccionaron dos primeros premolares libre caries y de restauraciones, se realizó el tallado con una convergencia hacia oclusal de $12^{\circ}$, $2 \mathrm{~mm}$ a nivel oclusal, $1,5 \mathrm{~mm}$ a nivel de las paredes axiales y a nivel del hombro $1 \mathrm{~mm}$ de espesor, para la línea de terminación chamfer y bisel. Luego fueron escaneados diseñados y fresados con el CAD/ CAM Ceramill ${ }^{\circledR}$ (Amann Girrbach AG, Koblach, Germany) (figura 1). Una vez obtenido el modelo maestro de Co-Cr, se realizó la impresión en un solo paso con silicona de adicción: silicona pesada Elite HD+Putty Soft Normal Set ${ }^{\circledR}$ (Zhermack Group, Badia Polesine, Italy) y silicona fluida Elite HD+Ligth Body Normal Set ${ }^{\circledR}$ (Zhermack Group, Badia Polesine, Italy). Luego se realizó el vaciado con yeso tipo IV extra duro Elite Master (Zhermark, Badia Polesine, Italia). Se obtuvó13 modelos de trabajo para cada técnica: CPCC en bisel, CPCC en chamfer, CPCI en bisel, CPCI en chamfer. Se procedió a realizar la delimitación con un lápiz de color rojo en la línea de terminación (8) y se aplicó un sellador de poros o endurecedor Die: Master Duo ${ }^{\circledR}$ (Renfert, Hilzingen, Alemania), luego se puso dos capas del espaciador Pico Fit ${ }^{\circledR}$ (Renfert, Hilzingen, Alemania) por encima de la línea de terminación, finalmente se aplicó una capa de aislante para cera Picosep ${ }^{\circledR}$ (Renfert, Hilzingen, Alemania) sobre la preparación. Como resultado se obtuvo las siguientes medidas: en la zona cervical $10 \mu \mathrm{m}$ y en el resto de la preparación tanto en las paredes axiales como en la parte oclusal $50 \mu \mathrm{m}(8)$. 
Para el patrón de cera de la cofia se empleó la técnica de cera por inmersión Renfert Hotty ${ }^{\circledR}$ (Renfert, Hilzingen, Alemania), con cera GEO-DIP® (Renfert, Hilzingen, Alemania). Para formar el collar cervical, se agregó cera con instrumentos de encerado PKT $\mathrm{N}^{\circ}$ 1. Se midió con un calibrador de cera, el cual fue de $0,5 \mathrm{~mm}$ de espesor. Por último, se readaptó el margen con la cera de márgenes StarWax $C \circledR$ (Dentaurum, Ispringen, Alemania). Obtenidos los patrones de cera, se añadió a cada uno, un bebedero de cera GEO ${ }^{\circledR}$ (Renfert, Hilzingen, Alemania. Luego la cofia se colocó en el formador de crisol nuevo, el cual fue revestido de yeso refractario Fórmula $1{ }^{\circledR}$ (WhipMix, Louisville, USA). Después, se colocó en el horno de calentamiento calibrado Mediterran 200 ( ) (Bego, Bremen, Alemania) por 30 minutos, a una temperatura inicial de $350^{\circ} \mathrm{C}$; aumentando gradualmente hasta una temperatura final de $900^{\circ} \mathrm{C}$, con la finalidad de calentar el revestimiento hasta que se evapore el patrón de cera (8). Para la técnica CPCC, se procedió a la fundición de la aleación de Co-Cr Cromoron Premium $C \circledR$ (DG Dental, Gornji Grad, Slovenia), con intervalo de fusión de $1304^{\circ} \mathrm{C}-1369^{\circ} \mathrm{C}$ y temperatura de colado de $1400^{\circ} \mathrm{C}-1480^{\circ} \mathrm{C}$, empleando un soplete calibrado M-3 con pico de lluvia (Tecnodent, Buenos Aires, Argentina). La llama tuvo forma de pincel y se acercó al crisol con el metal a una distancia de 5 $\mathrm{cm}$ cerciorándose que la zona útil o de reducción de la llama, esté en contacto con la aleación. Se mantuvo sobre el metal constantemente, hasta el cambio de estado sólido a líquido del metal. Luego, se trasladó el cilindro desde el horno hacia la centrífuga para colados previamente calibrada TECHINC@ (Técnica Dental, Bogotá, Colombia) para iniciar el movimiento de alta velocidad girando entre 300-3000 rpm alrededor de su eje, haciendo que el metal fundido ingrese al cilindro (8). Una vez obtenidas las cofias unitarias de Co-Cr, se evaluó la adaptación marginal de la cofia en el modelo maestro, a través de la inspección visual y el uso de la punta de un explorador.

En la técnica CPCI se realizó el mismo procedimiento de encerado, revestido y eliminación de cera anteriormente descrito pero, la diferencia es que no se usó el soplete para fundir el metal. Esta técnica empleó una máquina calibrada centrífuga compacta para colado con mecanismo de fusión por inducción eléctrica de gran potencia Fornax ${ }^{\circledR}$ T (BEGO GmbH, Bremen, Alemania) de 3600 VA y refrigeración integrada. Se precalentó el metal en la máquina durante 15 segundos, y se ingresó el cilindro de yeso refractario. Para ello, se configuró la máquina de inducción, para que suba la temperatura a $1200^{\circ} \mathrm{C}$, y luego de 25 segundos se observó que la aleación cambió de estado sólido a líquido. En ese momento, se activó la centrífuga, para que la aleación ingrese en el cilindro por acción de una fuerza al vacío (8).Terminado el enfriamiento de los colados, se realizó el desrevestido. Las cofias que no ingresaron fueron desgastadas internamente con una fresa de carburo-tungsteno de forma cónica, hasta observar un asentamiento adecuado. Una vez obtenidas las cofias unitarias de Co-Cr, se evaluó la adaptación marginal de la cofia en el modelo maestro, a través de la inspección visual y el uso de la punta de un explorador.

Se evaluó la adaptación marginal e interna usando la técnica de la réplica de silicona Fit Checker ${ }^{\mathrm{TM}}$ Advance ${ }^{\circledR}$ (GC Corporation, Tokyo, Japan) en el interior de la cofia, con la ayuda de una pistola de silicona utilizando una punta mezcladora con un tiempo de trabajo de $1 \mathrm{~min}$, posteriormente se asentó dicha cofia sobre el modelo maestro y se aplicó una presión constante de $15 \mathrm{~N}$, en una máquina de ensayo universal Instron CTM-5L ${ }^{\circledR} \quad$ (LG Electronics, Seúl, Korea) durante 1 min según el fabricante (8). A continuación se retiró la presión y la cofia fue removida del modelo maestro, conservando la película de silicona en el interior de ésta y seguidamente se inyectó la silicona VPS Elite HD+ Ligth Body Normal Set ${ }^{\circledR}$ (Zhermack Group, Badia Polesine, Italy) para obtener la copia del interior y conseguir la réplica de silicona. Luego de 48 horas cada réplica fue seccionada con una hoja de bisturí $\mathrm{N}^{\circ} 11$ en sentido vestíbulo-palatino y así se obtuvo dos fragmentos, uno mesial y otro distal, el fragmento que se escogió para ser medido será el mesial, luego se seccionó en sentido mesio-distal y el fragmento elegido para analizarlo, fue el fragmento palatino. Estos fragmentos fueron observados mediante un estéreomicroscopio calibrado Greenough Leica S8 APO ${ }^{\circledR}$ (Leica Biosystems GmbH, Wetzlar, Germany) a un aumento de 40X con el software Leica Application Suite LAS v3.4 ${ }^{\circledR}$ (Leica Biosystems $\mathrm{GmbH}$, Wetzlar, Germany). Estas medidas fueron a nivel de la zona cervical, axial y oclusal (figura 2).

La numeración "1" correspondió al corte en sentido vestíbulo-palatino donde el punto A1 fue el punto marginal vestibular y F1 fue el punto marginal palati- 
no, B1 y E1 representaron las zonas axiales, $\mathrm{C} 1$ y D1 la zona oclusal. La numeración "2" correspondió al corte en sentido mesio-distal donde el punto A2 fue el punto marginal mesial y F2 fue el punto marginal distal, B2 y E2 representaron las zonas axiales, C2 y D2 las zonas oclusales (figura 3 y figura 4) (8). Se realizó con el programa STATA versión 12. Con la información que fue recolectada se elaboró una base de datos, donde se evaluó los supuestos de normalidad con la prueba de Shapiro-Wilk. Se realizó el análisis univariado evaluando la media aritmética y desviación estándar. Para el análisis bivariado, se empleó para un grupo de resultados, la prueba t de Student si presentó distribución normal o en caso contrario, se realizó la prueba de U Mann-Whitney. El presente estudio utilizó un nivel de confianza del $95 \%$ y no hubo ningún tipo de conflicto de interés.

\section{RESULTADOS}

El estudio realizó la comparación in vitro, de la adaptación marginal e interna de cofias unitarias de aleación de Co-Cr, usando un tamaño muestral de 13 cofias unitarias para cada grupo, con un total de 52 cofias unitarias de aleación Co-Cr. En cada muestra obtenida, se realizaron 12 mediciones, divididas en seis: en sentido vestíbulo-palatino y mesio-distal, respectivamente.

La tabla 1 muestra los resultados de las discrepancias marginales en cofias metálicas unitarias de aleación Co-Cr, realizadas por la técnica CPCC con línea de terminación chamfer y bisel según tipo de corte y punto de medición. En la línea de terminación bisel los valores fueron para el corte vestíbulo-palatino en los puntos: (A1:27,17 $\pm 21,11 \mu \mathrm{m}, \mathrm{F} 1: 48,66 \pm 37,58$ $\mu \mathrm{m})$ y corte mesio-distal fueron (A2:62,16 $\pm 18,38 \mu \mathrm{m}$, F2:47,91 $\pm 16,77 \mu \mathrm{m})$. Para la línea de terminación chamfer los valores fueron mayores tanto en el corte vestíbulo-palatino fueron (A1:89,65 $\pm 58,39 \mu \mathrm{m}$, F1:92 $\pm 88,40 \mu \mathrm{m})$ como en el corte mesio-distal fueron $(\mathrm{A} 2: 91,72 \pm 67,13 \mu \mathrm{m}, \mathrm{F} 2: 97,66 \pm 50,59 \mu \mathrm{m})$.

La tabla 2 muestra los resultados de la discrepancia interna de cofias metálicas unitarias de aleación Co$\mathrm{Cr}$, obtenidos con la técnica CPCC sobre líneas de terminación chamfer y bisel según tipo de corte, zona y punto de medición. En la línea de terminación bisel en la zona axial para el corte vestíbulo-palatino, los valores fueron en los puntos: (B1:54,76 $\pm 21,02 \mu \mathrm{m}$,
$\mathrm{E} 1: 48,11 \pm 13,98 \mu \mathrm{m}), \quad \mathrm{y}$ en el corte mesio-distal fueron (B2:53,51 $\pm 24,95 \mu \mathrm{m}, \quad \mathrm{E} 2: 61,24 \pm 20,82$ $\mu \mathrm{m})$. En la zona oclusal los valores fueron para el corte vestíbulo-palatino $(\mathrm{C} 1: 125,40 \pm 41,84 \mu \mathrm{m}$, $\mathrm{D} 1: 157,08 \pm 45,04 \mu \mathrm{m})$, y en el corte mesio-distal $(C 2: 141,98 \pm 37,31 \mu \mathrm{m}, \mathrm{D} 2: 138,58 \pm 39,86 \mu \mathrm{m})$. Para la línea de terminación chamfer en la zona axial en el corte vestíbulo-palatino los valores fueron mayores (B1:60,09 $\pm 22,66 \mu \mathrm{m}, \mathrm{E} 1: 58,00 \pm 15,63 \mu \mathrm{m})$ pero menores en el corte mesio-distal fueron (B2:42,30 $\pm 23,32$ $\mu \mathrm{m}, \mathrm{E} 2: 52,62 \pm 26,16 \mu \mathrm{m})$. En la zona oclusal los valores fueron mayores tanto para el corte vestíbulo-palatino $(\mathrm{C} 1: 162,67 \pm 53,38 \mu \mathrm{m}, \mathrm{D} 1: 195,92 \pm 69,47 \mu \mathrm{m})$ como para el corte mesio-distal $(\mathrm{C} 2: 209,19 \pm 55,71 \mu \mathrm{m}$, D2:203,78 $62,94 \mu \mathrm{m})$

La tabla 3 muestra los resultados de la discrepancia marginal en cofias metálicas unitarias de aleación Co$\mathrm{Cr}$, realizadas por la técnica CPCI con línea de terminación chamfer y bisel según tipo de corte y punto de medición. En la línea de terminación bisel los valores fueron para el corte vestíbulo-palatino en los puntos: (A1:69,88 $\pm 19,74 \mu \mathrm{m}, \quad \mathrm{F} 1: 67,86 \pm 25,69 \mu \mathrm{m})$ $\mathrm{y}$ en el corte mesio-distal (A2:58,58 $\pm 14,67 \mu \mathrm{m}$, F2:64,02 $\pm 28,48 \mu \mathrm{m})$. Para la línea de terminación chamfer en el corte vestíbulo-palatino fue menor en el punto (A1:64,87 $\pm 27,36 \mu \mathrm{m})$ pero mayor en el punto $(\mathrm{F} 1: 72,13 \pm 22,62 \mu \mathrm{m})$ y en el corte mesio-distal fueron mayores (A2:93,43 $\pm 23,89 \mu \mathrm{m}, \mathrm{F} 2: 78,66 \pm 30,47 \mu \mathrm{m})$.

La tabla 4 muestra los resultados de la discrepancia interna de cofias metálicas unitarias de aleación Co$\mathrm{Cr}$, obtenidos con la técnica CPCI sobre líneas de terminación chamfer y bisel según tipo de corte, zona y punto de medición. En la línea de terminación bisel en la zona axial para el corte vestíbulo-palatino, los valores fueron en los puntos: (B1:40,90 $\pm 14,98 \mu \mathrm{m}$, E1: $39,18 \pm 10,15 \mu \mathrm{m})$, y en el corte mesio-distal (B2:51,94 $\pm 17,05 \mu \mathrm{m}, \mathrm{E} 2: 46,91 \pm 18,98 \mu \mathrm{m})$. En la zona oclusal los valores fueron para el corte vestíbulo-palatino $(\mathrm{C} 1: 217,85 \pm 64,30 \mu \mathrm{m}, \mathrm{D} 1: 224,44 \pm 96,35 \mu \mathrm{m})$ $\mathrm{y}$ en el corte mesio-distal $(\mathrm{C} 2: 164,83 \pm 53,59 \mu \mathrm{m}$, D2:166,68 $\pm 54,73 \mu \mathrm{m})$. En la línea de terminación chamfer, en la zona axial para el corte vestíbulo-palatino, los valores fueron mayores en los puntos: (B1:54,29 $\pm 24,92 \mu \mathrm{m}, \mathrm{E} 1: 54,29 \pm 18,29 \mu \mathrm{m})$ y menores en el corte mesio-distal (B2:37,98 $\pm 11,47 \mu \mathrm{m}$, E2:43,10 $\pm 14,38 \mu \mathrm{m})$. En la zona oclusal los valores fueron menores para el corte vestíbulo-palatino $(\mathrm{C} 1: 133,04 \pm 28,09 \mu \mathrm{m}, \mathrm{D} 1: 156,89 \pm 31,27 \mu \mathrm{m})$ у $\mathrm{ma}-$ 
yores en el corte mesio-distal $(\mathrm{C} 2: 173,33 \pm 25,56 \mu \mathrm{m}$, D2:172,93 $\pm 23,10 \mu \mathrm{m})$.

En la tabla 5 se comparó la adaptación marginal de las cofias metálicas unitarias de la técnicas CPCC y CPCI para las líneas de terminación chamfer y bisel. En la línea de terminación bisel, en el corte vestíbulo-palatino fue numéricamente mejor la adaptación marginal para la técnica de CPCC en comparación a CPCI pero solo fue estadísticamente significativo en el punto A1 ( $\mathrm{p}=0,0000)$, en el corte mesio-distal en A2 fue mejor la adaptación marginal para la CPCI y en $\mathrm{F} 2$ fue mejor la adaptación marginal para la CPCC, pero ambos puntos no tenían significancia estadística. En la línea de terminación chamfer, en el corte vestíbulo-palatino fue numéricamente mejor la adaptación marginal para la técnica de CPCI en comparación a CPCC pero no fue estadísticamente significativo en ningún punto, en el corte mesio-distal en $\mathrm{A} 2$ fue mejor la adaptación marginal para la CPCC y F2 fue mejor la adaptación marginal para la CPCI, pero ambos puntos no tenían significancia estadística.

En la tabla 6 se compararon la adaptación interna de las cofias metálicas unitarias de la técnica CPCC y CPCI para las líneas de terminación chamfer y bisel. En la línea de terminación bisel, en el corte vestíbulo-palatino en la zona axial fue numéricamente mejor la adaptación interna para la técnica de CPCI en comparación a CPCC pero no fue estadísticamente significativo en ningún punto. En la zona oclusal fue mejor la adaptación interna para la técnica de CPCC en comparación a CPCI y fue estadísticamente significativo en los puntos $\mathrm{C} 1(\mathrm{p}=0,0002)$ y $\mathrm{D} 1(\mathrm{p}=0,0355)$. En el corte mesio-distal en la zona axial, fue numéricamente mejor la adaptación interna para la técnica de CPCI en comparación a CPCC pero no fue estadísticamente significativo en ningún punto. En la zona oclusal, fue numéricamente mejor la adaptación interna para la técnica de CPCC en comparación a CPCI pero no fue estadísticamente significativo en ningún punto.
En la línea de terminación chamfer, en el corte vestíbulo-palatino en la zona axial, fue numéricamente mejor la adaptación interna para la técnica de CPCI en comparación a CPCC pero no fue estadísticamente significativo en ningún punto. En la zona oclusal, fue numéricamente mejor la adaptación interna para la técnica de CPCI en comparación a CPCC pero no fue estadísticamente significativo en ningún punto. En el corte mesio-distal en la zona axial, fue numéricamente mejor la adaptación interna para la técnica de CPCI en comparación a CPCC pero no fue estadísticamente significativo en ningún punto. En la zona oclusal fue numéricamente mejor la adaptación interna para la técnica de CPCI en comparación a CPCC pero solo fue estadísticamente significativo en el punto $\mathrm{C} 2$ de $(\mathrm{p}=0,0510)$. 
Tabla 1. Discrepancias marginales en cofias metálicas unitarias de aleación Co-Cr, confeccionadas por la técnica de la cera perdida colado por centrifugación convencional sobre líneas de terminación chamfer y bisel según el tipo de corte y punto de medición marginal.

\begin{tabular}{ccccccccc}
\hline $\begin{array}{c}\text { Línea de } \\
\text { terminación }\end{array}$ & Tipo de corte & $\begin{array}{c}\text { Punto } \\
\text { Marginal }\end{array}$ & Min & Max & Promedio & D.E. & Mediana & DIC \\
\hline \multirow{2}{*}{ Bisel } & Vestíbulo & A1 & 8,5 & 81,21 & 27,17 & 21,11 & 21,02 & 7,02 \\
& Palatino & F1 & 10,77 & 157,16 & 48,66 & 37,58 & 40,9 & 12,93 \\
& Mesio-Distal & A2 & 32,05 & 92,29 & 62,16 & 18,38 & 65,87 & 14,01 \\
& & F2 & 12,95 & 79,56 & 47,91 & 16,77 & 49,48 & 7,26 \\
\multirow{2}{*}{ Chamfer } & Vestíbulo & A1 & 32,77 & 219,94 & 89,65 & 58,39 & 57,93 & 76,08 \\
& Palatino & F1 & 26,18 & 296,2 & 92,00 & 88,40 & 56,92 & 60,95 \\
& Mesio-Distal & A2 & 23,02 & 224,83 & 91,72 & 67,13 & 63,5 & 89,51 \\
& & F2 & 38,62 & 202,63 & 97,66 & 50,59 & 84,42 & 64,78
\end{tabular}

Tabla 2. Discrepancias internas en cofias metálicas unitarias de aleación Co-Cr confeccionadas por la técnica de la cera perdida colado por centrifugado convencional sobre líneas de terminación chamfer y bisel según tipo de corte, zona y punto de medición interno.

\begin{tabular}{|c|c|c|c|c|c|c|c|c|c|}
\hline $\begin{array}{c}\text { Línea de } \\
\text { terminación }\end{array}$ & $\begin{array}{l}\text { Tipo de } \\
\text { corte }\end{array}$ & $\begin{array}{c}\text { Zona } \\
\text { interna }\end{array}$ & $\begin{array}{c}\text { Punto } \\
\text { Interno }\end{array}$ & Min & Max & Promedio & D.E. & Mediana & DIC \\
\hline \multirow{10}{*}{ Bisel } & \multirow{4}{*}{$\begin{array}{l}\text { Vestibulo } \\
\text { palatino }\end{array}$} & \multirow[t]{2}{*}{ Axial } & B1 & 34,31 & 104,64 & 54,76 & 21,02 & 45,79 & 25,88 \\
\hline & & & E1 & 21,92 & 73,43 & 48,11 & 13,98 & 46,23 & 9,81 \\
\hline & & \multirow[t]{2}{*}{ Oclusal } & $\mathrm{C} 1$ & 43,71 & 190,25 & 125,40 & 41,84 & 122,41 & 43,73 \\
\hline & & & D1 & 114,37 & 277,72 & 157,08 & 45,04 & 146,11 & 19,00 \\
\hline & \multirow{4}{*}{$\begin{array}{l}\text { Mesio } \\
\text { Distal }\end{array}$} & \multirow[t]{2}{*}{ Axial } & B2 & 30,58 & 99,58 & 53,51 & 24,95 & 39,88 & 44,36 \\
\hline & & & E2 & 16,94 & 94,20 & 61,24 & 20,82 & 64,97 & 26,00 \\
\hline & & \multirow[t]{2}{*}{ Oclusal } & $\mathrm{C} 2$ & 95,33 & 203,31 & 141,98 & 37,31 & 139,71 & 52,04 \\
\hline & & & D2 & 91,44 & 214,64 & 138,58 & 39,86 & 135,90 & 58,44 \\
\hline & \multirow{4}{*}{$\begin{array}{l}\text { Vestibulo } \\
\text { palatino }\end{array}$} & \multirow[t]{2}{*}{ Axial } & B1 & 29,32 & 100,43 & 60,09 & 22,66 & 54,61 & 14,43 \\
\hline & & & E1 & 35,42 & 79,57 & 58,00 & 15,63 & 61,03 & 21,24 \\
\hline \multirow{6}{*}{ Chamfer } & & Oclusal & $\mathrm{C} 1$ & 90,56 & 280,50 & 162,67 & 53,38 & 161,45 & 34,14 \\
\hline & & & D1 & 115,30 & 331,73 & 195,92 & 69,47 & 166,99 & 73,99 \\
\hline & \multirow{4}{*}{$\begin{array}{l}\text { Mesio } \\
\text { Distal }\end{array}$} & Axial & $\mathrm{B} 2$ & 11,43 & 80,17 & 42,30 & 23,32 & 35,76 & 27,92 \\
\hline & & & E2 & 24,26 & 114,87 & 52,62 & 26,16 & 44,45 & 22,71 \\
\hline & & \multirow[t]{2}{*}{ Oclusal } & $\mathrm{C} 2$ & 119,41 & 296,00 & 209,19 & 55,71 & 201.18 & 88,94 \\
\hline & & & D2 & 120,66 & 304,41 & 203,78 & 62,94 & 195.59 & 80,75 \\
\hline
\end{tabular}


Tabla 3. Discrepancias marginales en cofias metálicas unitarias de aleación Co-Cr, confeccionadas por la técnica de la cera perdida colado por centrifugación por inducción sobre líneas de terminación chamfer y bisel según el tipo de corte y punto de medición marginal.

\begin{tabular}{|c|c|c|c|c|c|c|c|c|}
\hline $\begin{array}{c}\text { Línea de } \\
\text { terminación }\end{array}$ & Tipo de corte & $\begin{array}{c}\text { Punto } \\
\text { Marginal }\end{array}$ & Min & Max & Promedio & D.E. & Mediana & DIC \\
\hline \multirow{5}{*}{ Bisel } & Vestibulo-Palatino & A1 & 31,32 & 98,05 & 69,88 & 19,74 & 72,92 & 22,13 \\
\hline & & $\mathrm{F} 1$ & 25,3 & 121,26 & 67,86 & 25,69 & 59,15 & 35,76 \\
\hline & Mesio Distal & A2 & 40,58 & 90,31 & 58,58 & 14,67 & 53,41 & 9,84 \\
\hline & & $\mathrm{F} 2$ & 25,61 & 120,69 & 64,02 & 28,48 & 60,85 & 24,71 \\
\hline & & A1 & 35,03 & 116,27 & 64,87 & 27,36 & 64,21 & 21,49 \\
\hline \multirow{3}{*}{ Chamfer } & Vestibulo-Palatino & $\mathrm{F} 1$ & 37,89 & 102,73 & 72,13 & 22,62 & 70,13 & 21,38 \\
\hline & Mesio Distal & A2 & 54,15 & 124,47 & 93,43 & 23,89 & 101,70 & 17,71 \\
\hline & & $\mathrm{F} 2$ & 38,62 & 125,07 & 78,66 & 30,47 & 69,29 & 21,30 \\
\hline
\end{tabular}

Tabla 4. Discrepancias internas en cofias metálicas unitarias de aleación Co-Cr, confeccionadas por la técnica de la cera perdida colado por centrifugado inducción sobre líneas de terminación chamfer y bisel según tipo de corte, zona y punto de medición interno.

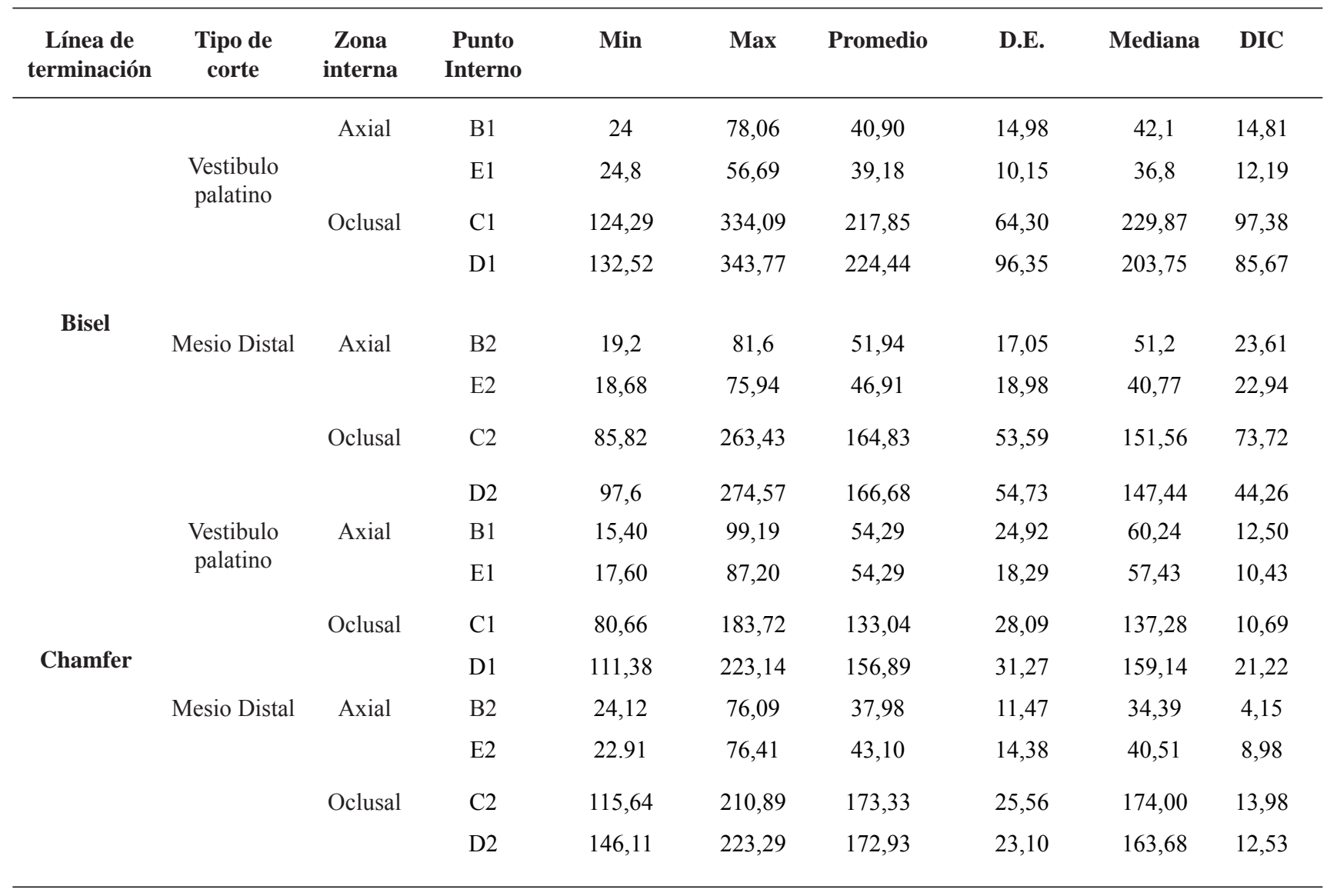


Tabla 5. Comparación de las adaptaciones marginales en cofias metálicas unitarias de aleación Co-Cr, confeccionadas por la técnica de la cera perdida colado por centrifugación convencional y por inducción con líneas de terminación chamfer y bisel, según tipo de corte y punto de medición marginal.

\begin{tabular}{|c|c|c|c|c|c|c|c|c|}
\hline \multirow[t]{2}{*}{$\begin{array}{l}\text { Líneas de } \\
\text { terminación }\end{array}$} & \multirow[t]{2}{*}{ Tipo de corte } & \multirow[t]{2}{*}{$\begin{array}{l}\text { Punto } \\
\text { Marginal }\end{array}$} & \multicolumn{2}{|c|}{$\begin{array}{l}\text { Centrifugación } \\
\text { convencional }\end{array}$} & \multicolumn{2}{|c|}{$\begin{array}{l}\text { Centrifugación por } \\
\text { inducción }\end{array}$} & \multirow[t]{2}{*}{$\begin{array}{l}\text { Diferencia de } \\
\text { medias }\end{array}$} & \multirow[t]{2}{*}{ Valor de $\mathrm{p}$} \\
\hline & & & Promedio & D.E. & Promedio & D.E. & & \\
\hline & Vestíbulo- & A1 & 27,17 & 21,11 & 69,88 & 19,74 & 19,74 & 5,2444 \\
\hline & & F1 & 48,66 & 37,58 & 67,86 & 25,69 & 25,69 & 1,5207 \\
\hline \multirow[t]{3}{*}{ Bisel } & Mesio-Distal & A2 & 62,16 & 18,38 & 58,58 & 14,67 & 14,67 & 0,9498 \\
\hline & & $\mathrm{F} 2$ & 47,91 & 16,77 & 64,02 & 28,48 & 28,48 & 1,7693 \\
\hline & Vestíbulo- & A1 & 89,65 & 58,39 & 64,87 & 27,36 & 27,36 & 24,78 \\
\hline \multirow[t]{3}{*}{ Chamfer } & & $\mathrm{F} 1$ & 92,00 & 88,40 & 72,13 & 22,62 & 22,62 & 19,86 \\
\hline & Mesio-Distal & A2 & 91,72 & 67,13 & 93,43 & 23,89 & 23,89 & $-1,70$ \\
\hline & & F2 & 97,66 & 50,59 & 78,66 & 39,47 & 39,47 & 19,01 \\
\hline
\end{tabular}

Prueba t student

significancia $\mathrm{p}<0,05$

U Mann-Whitney

Tabla 6. Comparación de las adaptaciones internas en cofias metálicas unitarias de aleación Co-Cr confeccionadas por la técnica de la cera perdida colado por centrifugación convencional y inducción con línea de terminación chamfer y bisel, según tipo de corte, zona y punto de medida interna.

\begin{tabular}{|c|c|c|c|c|c|c|c|c|c|}
\hline \multirow[t]{2}{*}{$\begin{array}{l}\text { Línea de } \\
\text { terminación }\end{array}$} & \multirow[t]{2}{*}{$\begin{array}{l}\text { Tipo de } \\
\text { corte }\end{array}$} & \multirow[t]{2}{*}{$\begin{array}{l}\text { Zona in- } \\
\text { terna }\end{array}$} & \multirow[t]{2}{*}{$\begin{array}{l}\text { Punto } \\
\text { interno }\end{array}$} & \multicolumn{2}{|c|}{$\begin{array}{l}\text { Centrifugación } \\
\text { convencional }\end{array}$} & \multicolumn{2}{|c|}{$\begin{array}{l}\text { Centrifugación por } \\
\text { inducción }\end{array}$} & \multirow[t]{2}{*}{ Diferencia de medias } & \multirow[t]{2}{*}{$\begin{array}{l}\text { Valor } \\
\text { de p }\end{array}$} \\
\hline & & & & Promedio & D.E. & Promedio & D.E. & & \\
\hline \multirow{8}{*}{ Bísel } & \multirow{3}{*}{$\begin{array}{l}\text { Vestíbulo } \\
\text { palatino }\end{array}$} & \multirow[t]{2}{*}{ Axial } & $\mathrm{B} 1$ & 54,76 & 21,02 & 40,90 & 14,98 & 13,86 & 0,0647 \\
\hline & & & E1 & 48,11 & 13,98 & 39,18 & 10,15 & 8,93 & 0,0746 \\
\hline & & \multirow[t]{2}{*}{ Oclusal } & $\mathrm{C} 1$ & 125,40 & 41,84 & 217,85 & 64,30 & $-92,45$ & 0,0002 \\
\hline & \multirow{5}{*}{$\begin{array}{l}\text { Mesio } \\
\text { Distal }\end{array}$} & & D1 & 157,08 & 45,04 & 224,44 & 96,35 & $-67,36$ & 0,0355 \\
\hline & & \multirow[t]{2}{*}{ Axial } & B2 & 53,51 & 24,95 & 51,94 & 17,05 & 1,57 & 0,8530 \\
\hline & & & E2 & 61,24 & 20,82 & 46,91 & 18,98 & 14,33 & 0,0791 \\
\hline & & \multirow[t]{2}{*}{ Oclusal } & $\mathrm{C} 2$ & 141,98 & 37,31 & 164,83 & 53,59 & $-22,85$ & 0,2192 \\
\hline & & & D2 & 138,58 & 39,86 & 166,68 & 54,73 & $-28,1$ & 0,1476 \\
\hline \multirow{8}{*}{ Chamfer } & \multirow{4}{*}{$\begin{array}{l}\text { Vestíbulo } \\
\text { palatino }\end{array}$} & \multirow[t]{2}{*}{ Axial } & B1 & 60,09 & 22,66 & 54,29 & 24,92 & 5,8 & 0,5405 \\
\hline & & & E1 & 58,00 & 15,63 & 54,29 & 18,29 & 3,71 & 0,5834 \\
\hline & & \multirow[t]{2}{*}{ Oclusal } & $\mathrm{C} 1$ & 162,67 & 53,38 & 133,04 & 28,09 & 29,63 & 0,0935 \\
\hline & & & D1 & 195,92 & 69,47 & 156,89 & 31,27 & 39,03 & 0,0833 \\
\hline & \multirow{4}{*}{$\begin{array}{l}\text { Mesio } \\
\text { Distal }\end{array}$} & \multirow[t]{2}{*}{ Axial } & $\mathrm{B} 2$ & 42,30 & 23,32 & 37,98 & 11,47 & 4,32 & 0,5568 \\
\hline & & & E2 & 52,62 & 26,16 & 43,10 & 14,38 & 9,52 & 0,2653 \\
\hline & & \multirow[t]{2}{*}{ Oclusal } & $\mathrm{C} 2$ & 209,19 & 55,71 & 173,33 & 25,56 & 35,86 & 0,0510 \\
\hline & & & D2 & 203,78 & 62,94 & 172,93 & 23,10 & 2,85 & 0,1179 \\
\hline
\end{tabular}

* Prueba t de Student

** U Mann-Whitney

significancia $\mathrm{p}<0,05$ 

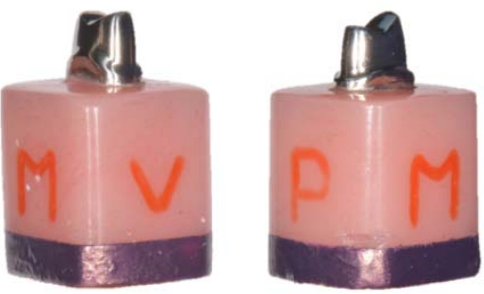

Figura 1. Modelos maestros en $\mathrm{Co}-\mathrm{Cr}$ con terminación en bisel y en chamfer.
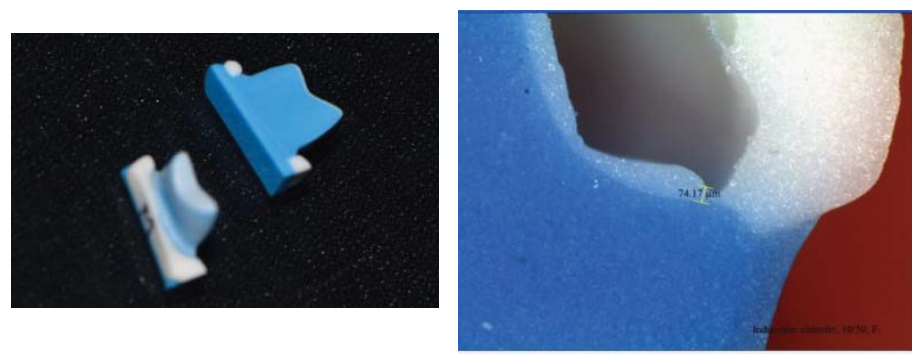

Figura 2. Cortes de la réplica de silicona y medición en $\mu \mathrm{m}$ de punto marginal.

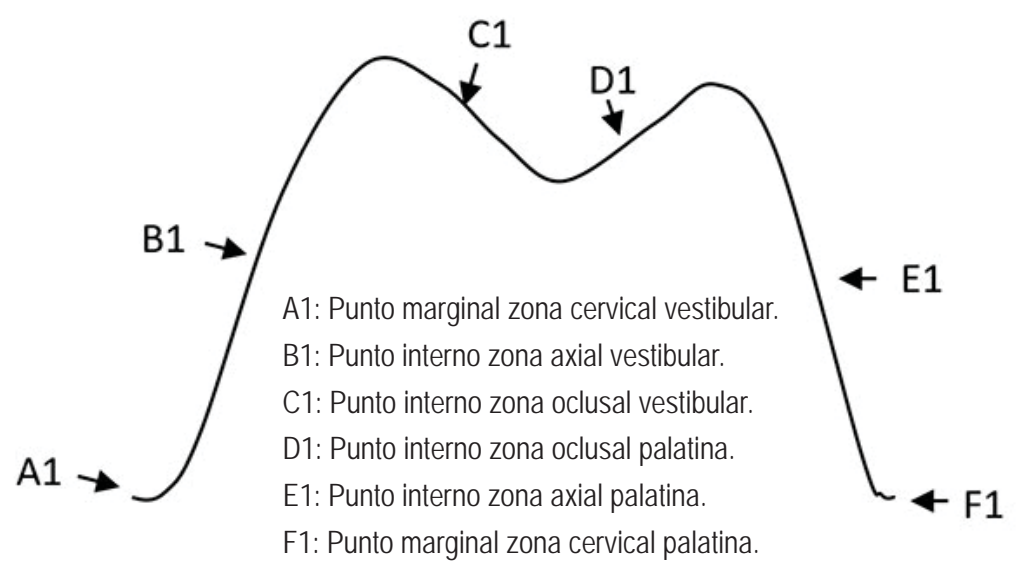

Figura 3. Corte vestíbulo-palatino

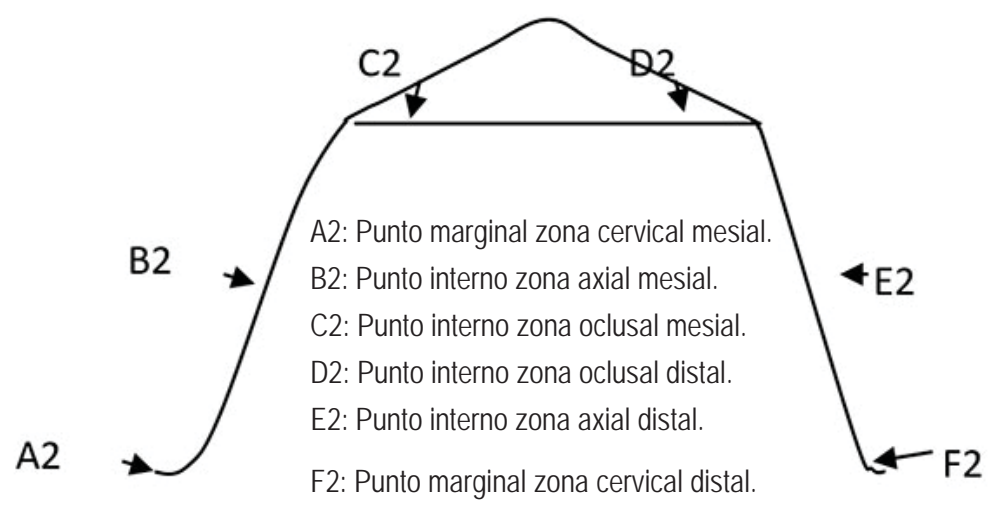

Figura 4. Corte mesio-distal 


\section{DISCUSIÓN}

Desde hace varios años, se ha venido discutiendo por diversos autores sobre cuál es la medida aceptable en micrómetros para la adaptación marginal, algunos autores proponen medidas entre 50 a $120 \mu \mathrm{m}(2,14,19)$ y sí nos referimos a la adaptación interna entre 50 y $350 \mu \mathrm{m}(7,8,20)$.

En el presente estudio, los resultados para la adaptación marginal usando la técnica CPCC y CPCI con dos líneas de terminación chamfer y bisel no fueron concluyentes pero mostraron medidas menores a las $120 \mu \mathrm{m}$. Siendo la técnica CPCC con la línea de terminación bisel la que tuvo mejor adaptación marginal, seguido por la técnica CPCI con la línea de terminación bisel, técnica CPCI con la línea de terminación chamfer y técnica CPCC con la línea de terminación chamfer. Al comparar la adaptación interna, usando la técnica CPCC y CPCI con dos líneas de terminación chamfer y bisel los resultados no fueron concluyentes pero mostraron medidas menores a las $350 \mu \mathrm{m}$. Para las zonas axiales la adaptación interna tuvo valores promedios menores a $62 \mu \mathrm{m}$ y para las zonas oclusales, tuvo valores promedios menores a $225 \mu \mathrm{m}$, en ambas técnicas la zona axial tuvo mejor adaptación interna que la zona oclusal. En el presente estudio, se pudo evidenciar que no existe diferencia estadísticamente significativa al comparar las técnicas CPCC y CPCI con las líneas de terminación chamfer y bisel, pero todos los valores promedio obtenidos fueron clínicamente aceptables, por lo tanto la hipótesis planteada no fue aceptada.

Se han propuesto varias líneas de terminación para coronas con cofias metálicas, sin embargo, existen pocos estudios que concluyan la superioridad de una con otra, en términos de adaptación marginal. Algunos estudios como Bottino et al., evaluó la adaptación marginal usando diferentes líneas de terminación, encontrando que el chamfer, obtiene mejores valores de adaptación marginal (1). Nemame et al., evaluó la adaptación marginal y oclusal en diferentes líneas de terminación entre ellas chamfer y bisel, encontrando que la terminación bisel proporciona un nivel de sellado superior que la terminación chamfer (21). Contrario a estos resultados Syu et al., y Rastogi, mostraron que el diseño de la línea de terminación no va a influenciar en la adaptación marginal $(22,23)$. Gómez-Cogolludo P et al., comparó la adap- tación marginal usando tres técnicas. Encontraron que el colado convencional ofreció mejores valores de adaptación, sin embargo, el estudio fue realizado con otros tipos de aleaciones (19). A su vez, Abad et al., en su estudio evaluaron la adaptación marginal usando cuatro técnicas convencionales. Concluyeron, que no hay diferencias significativas, ya que la discrepancia marginal en términos clínicos es aceptable para las cuatro máquinas (18). Requena comparo la adaptación marginal e interna, confeccionando cofias metálicas con dos sistemas CPCC Y CPCI con línea de terminación chamfer, sus resultados tampoco fueron concluyentes, no hubo diferencia significativa (20). El tipo de aleación utilizada fue de Co-Cr. Las aleaciones de metal base Ni-Cr, no se usaron en este estudio porque el Ni puede presentar una respuesta alérgica y el berilio está relacionado a consecuencias tóxicas $(2,24)$. Otro punto a considerar, fue el número de mediciones, ya que no existe un consenso y se basa en el criterio de cada investigador. En este estudio se usaron 12 puntos de medición en cada muestra, similar a los estudios realizados por Anusavise et al., y Jahanguiri et al., $(25,26)$.

Luego de haber analizado los resultados donde no se observa diferencia significativa, la decisión clínica entre una técnica y otra, dependerá de otros factores como la susceptibilidad a errores en el procedimiento, la contaminación del metal, la contaminación ambiental y sobretodo del personal, los cuales se evidencian en la técnica CPCC. Adicionalmente, se recomienda realizar investigaciones que permitan evaluar la adaptación marginal e interna mediante otras técnicas de fabricación, después del proceso de aplicación de la porcelana, con diferentes líneas de terminación, con diferente ángulo de convergencia, con otras aleaciones, etc; así como otras variables que puedan influir en la decisión clínica. Una limitación del estudio fue no haber usado un modelo maestro preparado digitalmente y no hubo conflicto de interés.

\section{CONCLUSIONES}

Las cofias metálicas unitarias de aleación de Co-Cr de la técnica CPCC con la línea de terminación bisel tuvo mejor adaptación marginal, seguido por la técnica CPCI con la línea de terminación bisel, técnica CPCI con la línea de terminación chamfer y técnica CPCC con la línea de terminación chamfer, pero al comparar estos resultados sólo son valores descripti- 
vos que no fueron concluyentes, porque en la mayoría de los puntos evaluados no existió diferencia estadísticamente significativa. Con respecto a la adaptación interna fue menor en las zonas axiales y mayor en las zonas oclusales para ambas técnicas y líneas de terminación, pero al comparar estos resultados sólo son valores descriptivos que no fueron concluyentes, ya que en la mayoría de los puntos evaluados no existió diferencia estadísticamente significativa.

\section{Correspondencia:}

Pedro Pantoja Borja.

Correo electrónico: pedro.pantoja.b@upch.pe

\section{REFERENCIAS BIBLIOGRÁFICAS.}

1. Bottino MA, Valandro LF, Buso L, Ozcan M. The influence of cervical finish line, internal relief, and cement type on the cervical adaptation of metal crowns. Quintessence Int. 2007; 38(7):425-32.

2. Roberts HW, Berzins DW, Moore BK, Charlton DG. Metal-ceramic alloys in dentistry: a review. J Prosthodont. 2009; 18(2):188-94.

3. Keith J. Ferro. Glossary of Prosthodontic Terms Committee of the Academy of Prosthodontics. J Prosthet Dent. 2017; 3(1):7-11.

4. Pulido F, Guerra c, Sánchez J, Giraldo H. Comparación de la adaptación marginal e interna de cofias coladas en dos sistemas de aleaciones. Rev Colomb Investig Odontol. 2014; 5(15):147-156.

5. Huang Z, Zhang L, Zhu J, Zhang X. Clinical marginal and internal fit of metal ceramic crowns fabricated with a selective laser melting technology. J Prosthet Dent. 2015; 113(6): 623-7.

6. Nawafleh N, Mack F, Evans J, Mackay J, Hatamleh M. Accuracy and reliability of methods to measure marginal adaptation of crowns and FDPs: a literature review. J Prosthodont. 2013; 22(5):419-28.

7. Land M, Hopp C. Survival rates of all-ceramic systems differ by clinical indication and fabrication method. J Evid Based Dent Pract. 2010; 10(1):37-8.

8. Loarte M. Adaptación de cofias metálicas confeccionadas con dos técnicas: cera pérdida colada por centrifugación convencional y fresado de bloque blando en CAD/CAM. Tesis para obtener el título profesional de especialista en Rehabilitación Oral. Lima: Universidad Peruana Cayetano Heredia; 2017. p. 68.

9. Compagni R, Faucher R, Yuodelis R. Effects of sprue design, casting machine, and heat source on casting porosity. J Prosthet Dent. 1984; 52(1): 41-45.

10. Hunter AJ, Hunter AR. Gingival margins for crowns: a review and discussion. Part II. Discrepancies and configurations. J Prosthet Dent. 1990; 64(6):63642.

11. Bishop K, Briggs P, Kelleher M. Margin desing for porcelain fused to metal restorations which extend onto the root. Br Dent J. 1996r; 180(5):177-84.

12. Vojdani M, Torabi K, Farjood E, Khaledi A. Comparison the marginal and internal fit of metal copings cast from wax patterns fabricated by $\mathrm{CAD} /$ CAM and conventional wax up techniques. J Dent 2013; 14(3):118-29.

13. Shillimburg H. Fundamentos esenciales en prótesis fija. $3^{\circ}$ Ed. Barcelona: Quintessence. 2006.

14. Cogolludo P, Suárez M, Peláez J, Lozano J. Influence of melting and casting methods and finish line design on the marginal discrepancy of nickel-chromiumtitanium Alloy Crowns. Int J Prosthodont. 2010; 23(5): 443-445.

15. Donovan TE, White LE. Evaluation of an improved centrifugal casting machine. J Prosthet Dent. 1985; 55(5): 609-12.

16. Compagni R, Faucher R, Yuodelis R. Effects of sprue design, casting machine, and heat source on casting porosity. J Prosthet Dent. 1984; 52(1): 41-5.

17. Abad J, Castro M, Durand E. Comparación de la adaptación marginal de cofias elaboradas en cuatro tipos de máquinas de colado. Rev Odontos. 2014; 43(4):7-14.

18. Gómez-Cogolludo P, Castillo-Oyagüe R, Lynch CD, Suárez-García MJ. Effect of electric arc, gas oxygen torch and induction melting techniques on the marginal accuracy of cast base-metal and noble metalceramic crowns. J Dent. 2013; 41(9): 826-31.

19. Requena S. Evaluación in vitro de la adaptación marginal e interna de cofias metálicas de aleación cobalto cromo ( $\mathrm{Co}-\mathrm{Cr}$ ) sobre una línea de terminación tipo chamfer fabricadas con dos técnicas de cera perdida coladas por centrifugación: convencional y por inducción. Tesis para obtener el título profesional de especialista en Rehabilitación Oral. Lima: Universidad Peruana Cayetano Heredia; 2018. 64p

20. Zelada CC. Comparación in vitro de las adaptaciones marginal e interna en cofias metálicas unitarias de aleación cobalto cromo (Co-Cr) realizadas sobre dos líneas de terminación: tipo chámfer y tipo bisel, fabricadas con las técnicas de la cera perdida colado por centrifugado convencional y de fusión selectiva por láser, diseño asistido por computadora/ manufactura asistida por computadora (CAD/CAM). Tesis para obtener el título profesional de especialista en Rehabilitación Oral. Lima: Universidad Peruana Cayetano Heredia; 2017. 80p

21. Nemane V, Akulwar R, Meshram S. The effect of various finish line configurations on the marginal seal and occlusal discrepancy of cast full crowns after 
cementation-an in-vitro study. J Clin Diagn Res 2015; 9(8): 18-21.

22. Syu J, Byrne G, Laub L, Land M. Influence of finishline geometry on the fit of crowns. Int J Prosthodont. 1993; 6(1):25-30.

23. Rastogi A, Kamble V. Comparative analysis of the clinical techniques used in evaluation of marginal accuracy of cast restoration using stereomicroscopy as gold standard. J Adv Prosthodont. 2011; 3(2):69-75.

24. Jabbari Y. Physico-mechanical properties and prosthodontic applications of $\mathrm{Co}-\mathrm{Cr}$ dental alloys: a review of the literatura. J Adv Prosthodont 2014; 6 (2):138-45.

25. Anusavice KJ, Phillips RW, Shen C, Rawls HR. Phillips' Science of Dental Materials. 12th ed. St. Louis: Elsevier/Saunders; 2013.

26. Jahangiri L, Wahlers C, Hittelman E, Matheson P. Assessment of sensitivity and specificity of clinical evaluation of cast restoration marginal accuracy compared to stereomicroscopy. J Prosthet Dent. 2005; 93(2):138-42.

Recibido: 18-11-2018

Aceptado: 15-03-2019 